\title{
Hyperexcitability in Spinal WDR Neurons following Experimental Disc Herniation Is Associated with Upregulation of Fractalkine and Its Receptor in Nucleus Pulposus and the Dorsal Root Ganglion
}

\author{
Daniel Pitz Jacobsen, ${ }^{1}$ Aurora Moen, ${ }^{1,2}$ Fred Haugen, ${ }^{1}$ and Johannes Gjerstad ${ }^{1,2}$ \\ ${ }^{1}$ National Institute of Occupational Health, Oslo, Norway \\ ${ }^{2}$ Department of Biosciences, University of Oslo, Norway
}

Correspondence should be addressed to Daniel Pitz Jacobsen; dpja@stami.no

Received 9 August 2016; Revised 11 November 2016; Accepted 29 November 2016

Academic Editor: David A. Hart

Copyright (C) 2016 Daniel Pitz Jacobsen et al. This is an open access article distributed under the Creative Commons Attribution License, which permits unrestricted use, distribution, and reproduction in any medium, provided the original work is properly cited.

Introduction. Lumbar radicular pain following intervertebral disc herniation may be associated with a local inflammatory response induced by nucleus pulposus (NP) cells. Methods. In anaesthetized Lewis rats, extracellular single unit recordings of wide dynamic range (WDR) neurons in the dorsal horn and qPCR were used to explore the effect of NP application onto the dorsal nerve roots (L3-L5). Results. A clear increase in C-fiber response was observed following NP conditioning. In the NP tissue, the expression of interleukin-1 $\beta$ (IL-1 $\beta$ ), colony stimulating factor 1 (Csf1), fractalkine (CX3CL1), and the fractalkine receptor CX3CR1 was increased. Minocycline, an inhibitor of microglial activation, inhibited the increase in neuronal activity and attenuated the increase in IL-1 $\beta$, Csf1, CX3L1, and CX3CR1 expression in NP tissue. In addition, the results demonstrated an increase in the expression of TNF, CX3CL1, and CX3CR1 in the dorsal root ganglions (DRGs). Conclusion. Hyperexcitability in the pain pathways and the local inflammation after disc herniation may involve upregulation of CX3CL1 signaling in both the NP and the DRG.

\section{Introduction}

Earlier studies show that leakage of nucleus pulposus (NP) out of the disc after intervertebral disc herniation is associated with a local release of many substances including interleukin$1 \beta$ (IL-1 $\beta$ ), tumor necrosis factor (TNF), and colony stimulating factor 1 (Csf1) [1-4]. Such cytokines released after intervertebral disc herniation may induce increased neuronal excitability in the primary afferent nerve fibers and reduced axonal speed of conduction and may also promote nerve injury $[1,5,6]$. Thus, intervertebral disc herniation has adverse effects on the sensory pathways.

Peripheral inflammation in animals may be associated with activation of satellite glial cells in the dorsal root ganglion (DRG) [7]. Nerve injury and release of inflammatory substances may also activate microglia in the spinal cord [8-10]. For instance, previous data show that soluble fractalkine (CX3CL1) activates glia and leukocytes through its receptor CX3CR1 [11, 12]. Furthermore, spinal upregulation of CX3CL1 may be associated with mechanical allodynia and thermal hyperalgesia [13]. Thus, one important inflammatory molecule possibly influencing the sensory pathways may be CX3CL1.

Evidence exists that the local inflammation associated with disc herniation as well as microglial activation is dependent on phosphorylation of MAPK p38 [14, 15]. Thus, drugs targeting this kinase may attenuate inflammatory pain $[10,16,17]$. Here, in an animal model mimicking clinical intervertebral disc herniation, we investigate the effect of NP on neuronal activity in the spinal cord and examine the local gene expression. We hypothesize that the interaction between NP cells and neuronal tissue may induce a local upregulation 
of CX3CL1 signaling and increased nociceptive activity in the pain pathways.

\section{Materials and Methods}

2.1. Animals and Surgery. A total of 87 adult inbred female Lewis rats (170-215 g) were used in the present study. Female rats were preferred because allergens are more potent in males. Before use, all rats were initially sedated with isoflurane gas (Baxter International Inc., USA) followed by intraperitoneal administration of $250 \mathrm{mg} / \mathrm{mL} \sim 2.1 \mathrm{~g} / \mathrm{kg}$ body weight urethane (Sigma-Aldrich Co., Germany). Absence of hind paw withdrawal and ear wriggling to pinch indicated adequate surgical anesthesia. The rat's core temperature was maintained at $36-37^{\circ} \mathrm{C}$ by a feedback heating pad.

To expose the sciatic nerve, a $1-2 \mathrm{~cm}$ incision was made above the pelvic girdle and a bipolar silver hook electrode was placed proximal to the main branches of the sciatic nerve for electrical stimulation. As previously described [1], a laminectomy, with a $1 \mathrm{~mm}$ lateral expansion on the left side for the application of NP, was performed at vertebrae Th13-L1, corresponding to the spinal cord segments L3-S1. The vertebral column was rigidly fixed by clamps rostral and caudal to the exposed spinal cord segments. The meninges, that is, dura mater and arachnoidea, were punctured by a cannula and carefully removed by two tweezers. The animals were euthanized by an overdose of pentobarbital.

2.2. In Vivo Electrophysiology. A parylene-coated tungsten microelectrode with impedance 2-4 M $\Omega$ (Frederick Haer \& Co., USA) was lowered vertically into the left dorsal horn of the spinal cord by an electrically controlled micromanipulator (Märzhäuser Wetzlar GmbH \& Co. KG, Germany). The spinal cord segments L3-S1 were identified by the neuronal response to left hind paw finger tapping and pinching. Extracellular single cell recordings of wide dynamic range (WDR) neurons were performed at depths of 50$700 \mu \mathrm{m}$ from the surface of the spinal cord. Only one cell was studied in each animal. The recorded signals from the headstage were amplified $(\times 5000)$ with an AC preamplifier, band-pass filtered with half amplitude cut of $500-1250 \mathrm{~Hz}$ (NeuroLog by Digitimer Ltd, UK), digitalized with interface CED Micro1401, and displayed on a computer screen by the software CED Spike 2.2 (Cambridge Electronic Design, UK). The sampling frequency was $20 \mathrm{kHz}$.

The software Spike 2.2 was also used to control the electrical stimuli frequency given to the sciatic nerve by the hook electrode, while a pulse buffer connected to a stimulus isolator unit (NeuroLog System, Digitimer Ltd, UK) controlled the stimuli intensities. Single cell recordings were ensured by examining spike shape and amplitude. Spikes 50$300 \mathrm{~ms}$ after stimulus were defined as $\mathrm{C}$-fiber responses. The C-fiber threshold was defined at the beginning of each experiment as the lowest stimulus intensity capable of eliciting a single visible spike in the defined C-fiber timeframe. Every fourth minute a single test stimuli ( $2 \mathrm{~ms}$ rectangular pulse, $1.5 x$ C-fiber threshold) was applied to the sciatic nerve. Six stable recordings varying less than $20 \%$ served as a baseline for the subsequent experiments. Only cells with baseline responses of 5-20 spikes were included in the study.

To study the effect of NP conditioning on neuronal activity, NP was harvested from 3 to 4 caudal intervertebral discs of a genetically identical donor rat and applied $0.5-2 \mathrm{~mm}$ caudally to the recording electrode, covering the incoming left dorsal nerve roots. Minocycline (MC, M9511, SigmaAldrich Co., Germany) was chosen to target MAPKp38 in the glial cells. $\mathrm{MC}$ was dissolved in $0.9 \% \mathrm{NaCl}$, diluted to a concentration of $5 \mu \mathrm{g} / \mu \mathrm{L}$, and topically administered onto the nerve roots $(25 \mu \mathrm{L}$ before and $25 \mu \mathrm{L}$ after NP application). Four series of electrophysiological experiments were performed: (I) NP conditioning $(n=7)$, (II) NP conditioning together with $\mathrm{MC}(n=7)$, (III) $\mathrm{MC}(n=5)$, and (IV) $\mathrm{NaCl}$ control $(n=5)$. The C-fiber response was followed for 180 minutes after conditioning. In addition, 14 animals were used as donor rats.

2.3. Parallel NP Gene Expression Experiments. In order to investigate changes in the expression of our candidate genes in NP tissue, four series of experiments were performed and defined as (I) NP, (II) NP+MC, (III) $\mathrm{NP}^{\text {fat }}$, and (IV) $\mathrm{NP}^{\text {native }}$. The tissue in the NP group $(n=7)$ had been in contact with the dorsal nerve roots for 180 minutes, as had the tissue in the NP+MC group $(n=7)$ together with minocycline. Tissue samples for these two groups were harvested directly following the electrophysiological experiments described above. The $\mathrm{NP}^{\text {fat }}$ group $(n=9)$, which was applied onto neck fat tissue for 180 minutes, served as a control group to investigate whether any possible gene expression changes in the NP group were caused by the tissue-specific properties of the nerve roots or surrounding tissues. $\mathrm{NP}^{\text {native }}$ tissue ( $n=10)$ was frozen directly after isolation from the caudal intervertebral discs of the donor rats and served as control. In addition, 9 extra animals were used for the fat controls.

\subsection{Ipsi- and Contralateral Dorsal Root Ganglion Gene Expres-} sion. We also wanted to investigate the gene expression changes in the DRG following experimental disc herniation. Thus, lumbar DRG L3-L5, giving input to TH13 and L1 through their dorsal nerve roots, were dissected out and frozen on liquid nitrogen. The DRG isolation protocol has been described elsewhere [18]. Two series of DRG experiments were performed: NP $(n=7)$ and native $(n=7)$. In both groups, a laminectomy exposing the dorsal nerve roots was performed three hours before isolation of DRG. In the NP group, NP tissue from the caudal intervertebral discs of a donor rat was applied onto the left dorsal nerve roots immediately after the laminectomy. In total, 7 donor rats were used for DRG gene expression experiments. Both the left and the right dorsal root ganglia were isolated. Left L3, L4, and L5 and right L3, L4, and L5 were pooled separately before gene expression analyses.

2.5. $q$ PCR. As previously described [19], total RNA was extracted from frozen $\left(-80^{\circ} \mathrm{C}\right) \mathrm{NP}$ and $\mathrm{DRG}$ tissue by the TRIzol reagent (Life technologies, Inc., Rockville, Maryland, USA), chloroform (Sigma-Aldrich, St. Louis, MO, USA), and isopropanol (Merck, Darmstadt, Germany). RNA 
TABle 1: Primer sequences.

\begin{tabular}{|c|c|c|c|c|}
\hline Primer & Sequence $5^{\prime} \rightarrow 3^{\prime}$ & Bp & $\% \mathrm{GC}$ & $\mathrm{Tm}^{\circ} \mathrm{C}$ \\
\hline IL1 $\beta$ forward & CGT GGA GCT TCC AGG ATG AG & 20 & 60.0 & 59.4 \\
\hline IL1 $\beta$ reverse & CGT CAT CAT CCC ACG AGT CA & 20 & 50.0 & 59.1 \\
\hline TNF forward & GCC ACC ACG CTC TTC TGT CTA & 21 & 57.1 & 59.1 \\
\hline TNF reverse & TGA GAG GGA GCC CAT TTG G & 19 & 57.9 & 59.6 \\
\hline Csf1 forward & GGG AAT GGA CAC CTA CAG ATT TTG & 24 & 45.8 & 59.6 \\
\hline Csf1 reverse & AAA TTT ATA TTC GAT CAG GCA TGCA & 25 & 32.0 & 59.7 \\
\hline CX3CL1 forward & TTG CAC AGC CCA GAT CAT TC & 21 & 47.6 & 54.3 \\
\hline CX3CL1 reverse & CTG CGC TCT CAG ATG TAG GAA A & 22 & 50.0 & 55.7 \\
\hline CX3CR1 forward & GTG GCC TTT GGG ACC ATC T & 19 & 57.9 & 56.7 \\
\hline CX3CR1 reverse & CCA CCA GAC CGA ACG TGA A & 19 & 57.9 & 56.6 \\
\hline$\beta$-actin forward & CTA AGG CCA ACC GTG AAA AGA & 21 & 47.6 & 58.0 \\
\hline$\beta$-actin reverse & ACA ACA CAG CCT GGA TGG CTA & 21 & 52.4 & 59.2 \\
\hline
\end{tabular}

was reversibly transcribed by aid of the first-strand cDNA Synthesis Kit for reverse-transcriptase polymerase chain reaction (RT-PCR) (AMV) (Roche Diagnostic, Mannheim, Germany). The qPCR analysis was then performed in two parallels on a StepOnePlus qPCR machine (Applied Biosciences, USA). Primers were designed using Primer Express 2.0 (Applied Biosystems, California, USA) and checked for specificity by performing a BLAST search. Effort was made to design primers without nonspecific binding (the melting curves indicated no biproducts). For more details about the primers (Risskov, Denmark), see Table 1. Target genes were normalized to $\beta$-actin (internal reference).

2.6. Statistics. The spinal nociceptive activity, that is, the $\mathrm{C}$ fiber response, was presented as percent of baseline. The 6 baseline recordings were converted to 2 baseline values (each an average of 3 consecutive responses), and the 45 postbaseline recordings were converted to 9 values (each an average of 5 consecutive responses). To examine the effect of the four types of intervention, NP, NP+MC, MC, and control, on spinal nociceptive response, an analysis of variance, repeated-measure design (rmANOVA), was performed. Because the assumption of sphericity was not met, the degrees of freedom were corrected using a GreenhouseGeisser correction. The average C-fiber responses from 60 to 180 minutes after conditioning were compared using one-way ANOVA and Tukey's honestly significant difference (HSD) post hoc comparisons. Regarding the gene expression, fold change values for each sample were defined by the expression of the target gene normalized to the expression of the reference gene $\beta$-actin and the level of the corresponding native tissue. Group means of NP expression values were compared using one-way ANOVA and Tukey's HSD post hoc comparisons, while DRG expressions were compared with an unpaired Student's $t$-test. Statistical analyses were performed by use of SPSS 21 (IBM SPSS inc., USA). A $P$ value $<0.05$ was set as the level of statistical significance. Data are given as representative examples and means \pm SEM.

\section{Results}

We here report that IL-1 $\beta$ (red), TNF (green), Csf1 (blue), but also CX3CL1 (brown), and CX3CR1 (yellow) were expressed in the native NP tissue (Figure 1(a)). Moreover, NP applied onto the dorsal nerve roots induced a rapid increase in the C-fiber response (Figure 1(b)), but this was in most cases blocked by minocycline (Figure 1(c)). The observed increase in C-fiber response was evident already 10-20 minutes after NP administration (Figure 2(a)), whereas no clear increase in the C-fiber response was seen following application of NP together with minocycline (Figure 2(b)). Application of minocycline alone caused a short lasting decrease in $\mathrm{C}$ fiber response (Figure 2(c)). No clear changes in the C-fiber response were observed in the vehicle control experiments (Figure 2(d)).

The average C-fiber response 60-180 minutes after NP conditioning was $136.1 \% \pm 13.9$ of baseline, but after NP conditioning together with minocycline only $99.9 \% \pm 6.5$ of baseline. Moreover, the average $\mathrm{C}$-fiber response after minocycline alone was $92.0 \% \pm 8.6$ of baseline, and in the corresponding vehicle control $91.7 \% \pm 7.4$ of baseline (Figure 2(e)). The C-fiber response after conditioning with NP alone was significantly higher than the C-fiber response after conditioning with NP plus minocycline, minocycline alone or in the corresponding vehicle control.

Moreover we observed a clear increase in the expression of IL1 $\beta$ in NP samples, but no change in the expression of TNF, and a minor increase in the expression of macrophage colony stimulating factor Csf1 180 minutes after application onto the nerve roots (fold expression: IL- $1 \beta ; 13.47 \pm 3.03$, TNF $2.89 \pm 2.08$, Csfl; $3.13 \pm 1.21)$. Notably, a significant increase in the gene expression of CX3CL1 and its cognate receptor CX3CR1 was also observed in the NP tissue (fold expression: CX3CL1; $7.32 \pm 1.89$, CX3CR1; $7.32 \pm 1.89$ ).

Interestingly, the NP-induced changes in expression were almost completely blocked when NP was applied together with minocycline, the inhibitor of glial cells and macrophage activation (fold expression: IL1 $\beta ; 5.19 \pm 2.08$, TNF; $1.55 \pm 0.43$, Csf1; $0.92 \pm 0.15$, CX3CL1; $2.01 \pm 0.51$, CX3CR1; $1.95 \pm 0.45)$. 


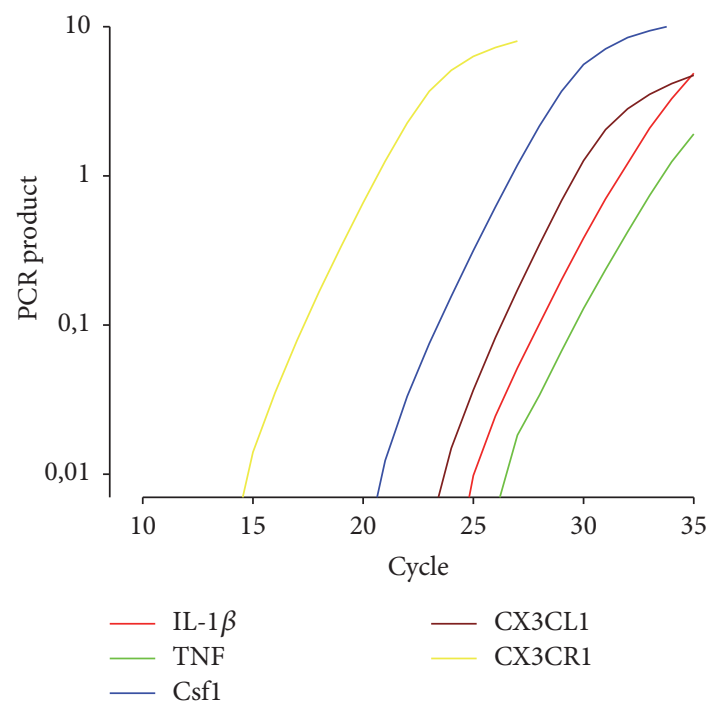

(a)

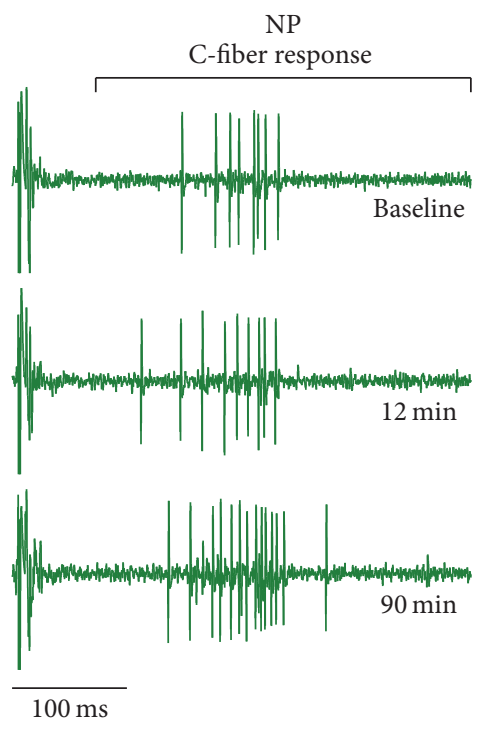

(b)

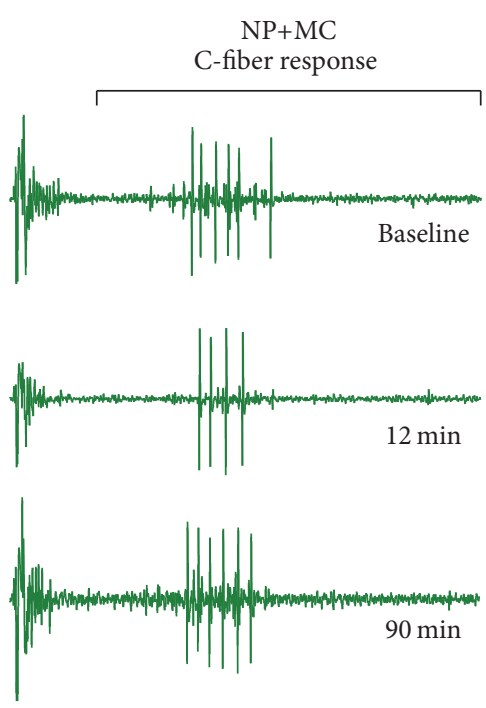

(c)

Figure 1: (a) Examples of qPCR amplification plots demonstrating the presence IL-1 $\beta$ (red), TNF (green), Csf1 (blue), CX3CL1 (brown), and CX3CR1 (yellow) in native NP tissue. (b) Examples of single cell recordings at baseline, 12 minutes and 90 minutes after nucleus pulposus conditioning alone. (c) Examples of single cell recordings at baseline, 12 minutes and 90 minutes after nucleus pulposus conditioning together with minocycline.

No clear changes in expression were observed in the corresponding fat control experiments where the NP was applied onto the intrascapular adipose tissue (fold expression: IL1 $\beta$; $3.68 \pm 0.54$, TNF; $1.99 \pm 0.32$, Csf1; $1.53 \pm 0.25$, CX3CL1; $2.51 \pm$ 0.33 , CX3CR1; $2.66 \pm 0.47$ ). Thus, the increase in expression, which was dependent on the contact between the NP tissue and the dorsal nerve roots, was attenuated by minocycline (Figures 3(a)-3(e)).

After 3 hours, NP applied onto the left dorsal nerve roots induced an ipsilateral increase in the expressions of TNF, CX3CL1, and CX3CR1 in the DRGs (fold expression in ipsilateral DRGs: TNF; $1.91 \pm 0.56$, CX3CL1; $1.88 \pm 0.13$, CX3CR1; $1.86 \pm 0.26$; fold expression in contralateral DRGs: TNF; $1.47 \pm 0.56$, CX3CL1; $1.23 \pm 0.27$, CX3CR1; $1.33 \pm$ 0.47 ) (Figures 4(a)-4(e)). Although NP was applied 5-15 mm proximal to the DRG, this seemed to have a clear effect on the DRG cells. The fold expressions of IL1- $\beta$ and Csf1, however, were not increased (ipsilateral DRGs: IL-1 $\beta ; 1.08 \pm 0.16$, Csf1; $1.03 \pm 0.07$; contralateral DRGs: IL-1 $\beta ; 1.10 \pm 0.28$, Csfl; $1.08 \pm 0.09)$.

\section{Discussion}

Our results showed that application of NP onto the dorsal nerve roots within minutes increased the $\mathrm{C}$-fiber response in the dorsal horn. The changes in gene expression in the DRG suggest that the primary afferent nerve fibers may be affected by NP. In addition, proinflammatory cytokines released near the dorsal nerve roots could diffuse to the dorsal horn and affect postsynaptic cells and spinal microglia. Thus, the increase in excitability at the spinal level may be due to direct effects of NP on the primary nerve fibers, but also secondary changes induced by molecules from NP in postsynaptic WDR neurons. Previous findings indicate that the rapid effects of NP are induced by IL- $1 \beta$ and TNF action on dorsal horn lamina II ion channels and suppressed spinal GABA and glycine-induced currents [20,21]. The effect of $\mathrm{NP}$ on nociceptive signaling has also been demonstrated in behavioral studies [22].

Previous observations show that application of NP onto the dorsal nerve roots may affect the expression of cytokines like IL- $1 \beta$ and TNF in the NP tissue [1]. In accordance with these earlier findings, the present data show a significant upregulation of IL-1 $\beta$ after application of NP onto the dorsal nerve roots in the NP tissue. However, in contrast to the observation of our earlier report, no clear upregulation of TNF was observed in the NP tissue this time. On the other hand, the present data showed a significant upregulation of TNF in the DRG.

Anyway, several lines of evidence suggest that IL-1 $\beta$ and TNF, but also Csfl, may be upregulated in NP or surrounding tissue following disc herniation $[1,3,4,23]$. Therefore, it seems likely that persistent hyperexcitability in the pain pathways after disc herniation is associated with a local inflammatory process including activation of macrophages close to the nerve roots. Moreover, previous data show that IL-1 $\beta[24,25]$, Csf1 [26-28], and CX3CL1 $[22,29,30]$ can induce M1 microglia activation. For the first time, however, we showed that application of NP onto the dorsal nerve roots also increased the gene expression of CX3CL1 and CX3CR1 in the NP cells and in the ipsilateral DRGs. These observations suggest that local upregulation of IL-1 $\beta$, Csf1, and CX3CL1 following disc herniation may activate microglia and induce pain [21]. Interestingly, no change in gene expression was 


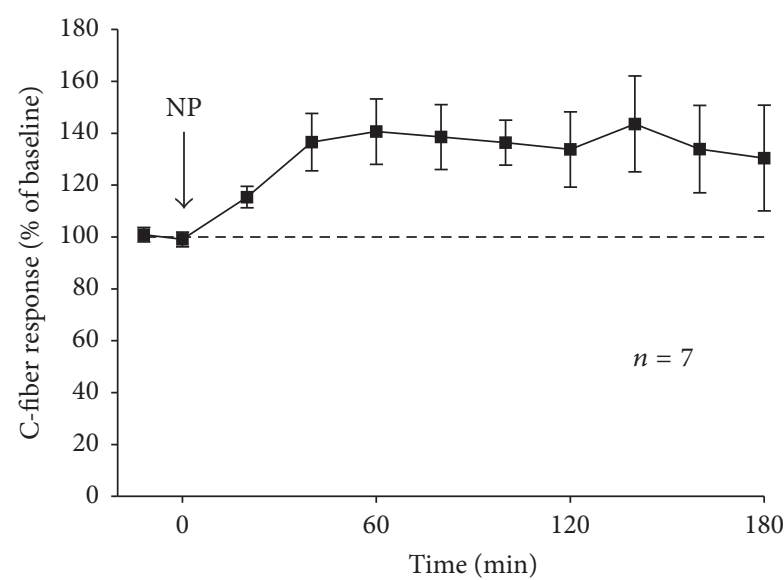

(a)

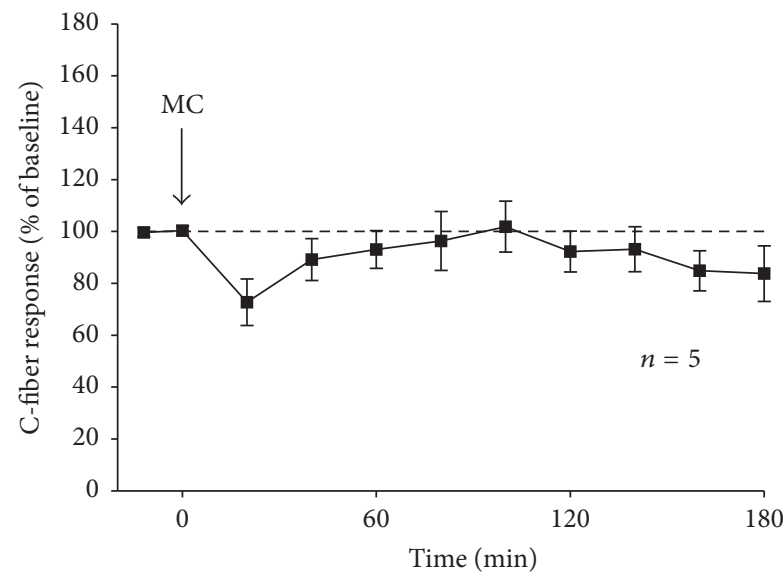

(c)

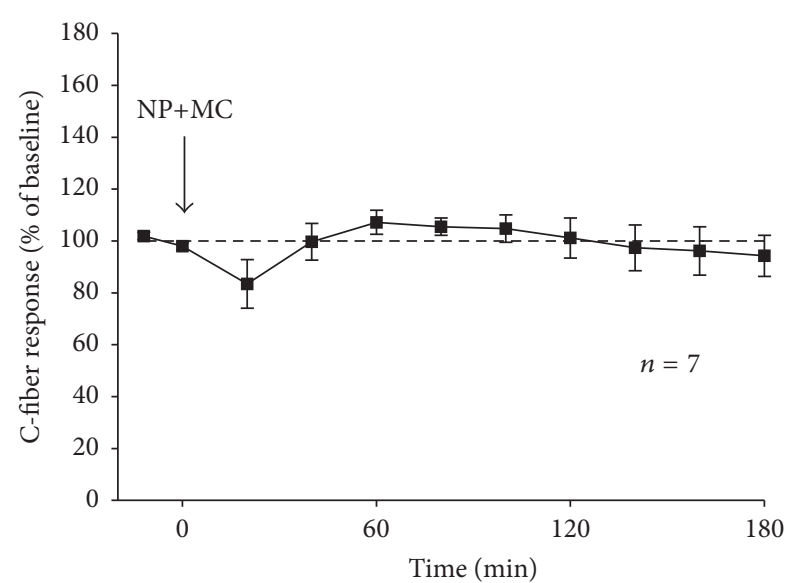

(b)

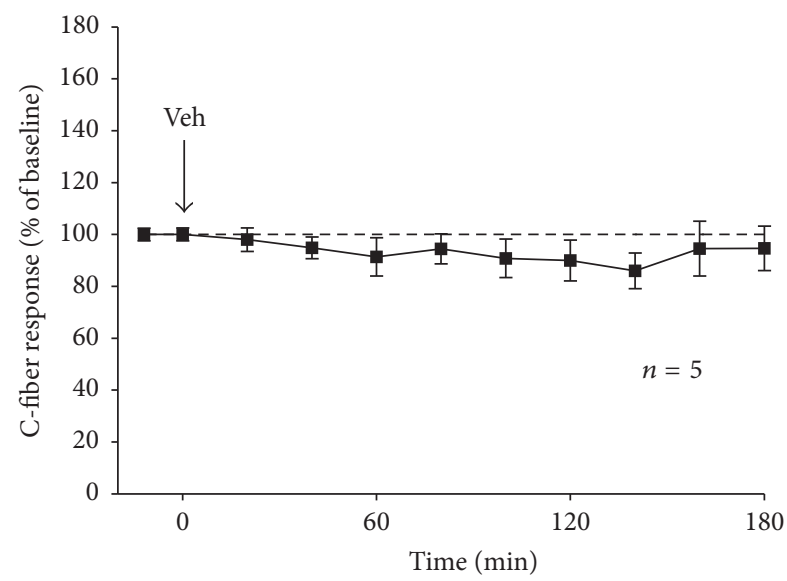

(d)

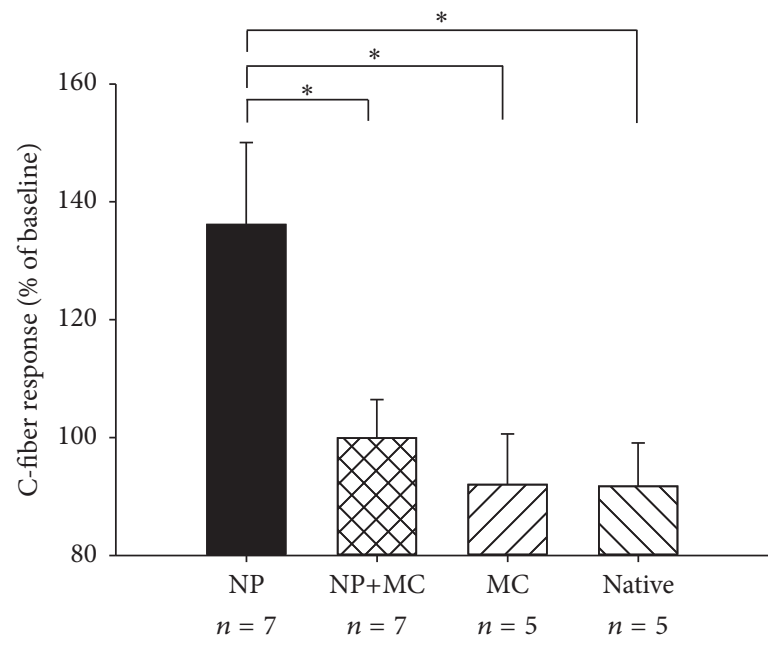

(e)

Figure 2: C-fiber response in percent of baseline after application of (a) NP (NP), (b) NP and minocycline (NP+MC), (c) minocycline (MC), and (d) Vehicle (Veh). (e) The mean value 60 to 180 minutes after baseline in the four groups. $P=0.018$, rmANOVA, four groups; NP, $\mathrm{NP}+\mathrm{MC}, \mathrm{MC}$, Veh. ${ }^{*} \mathrm{P}<0.05$, one-way ANOVA, Tukey's post hoc test. 


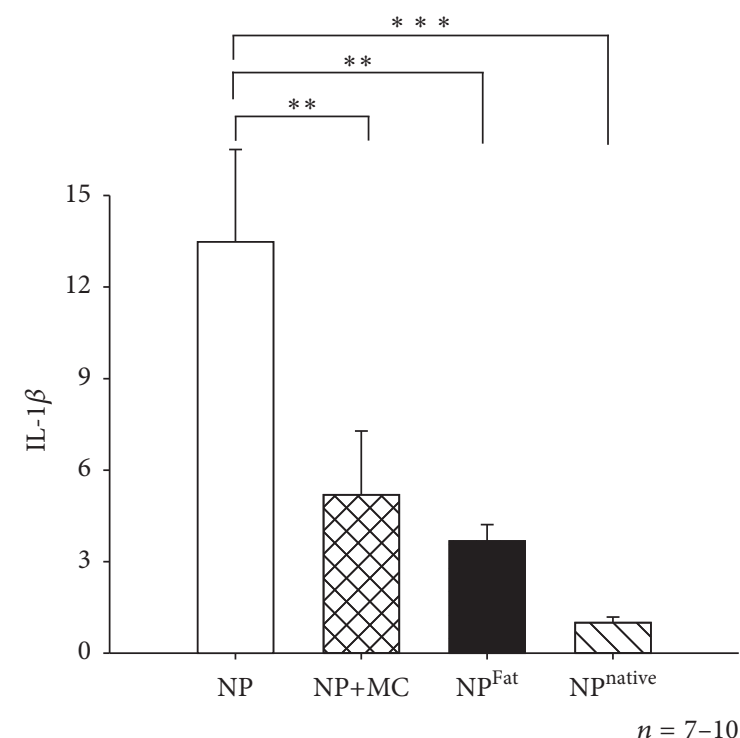

(a)

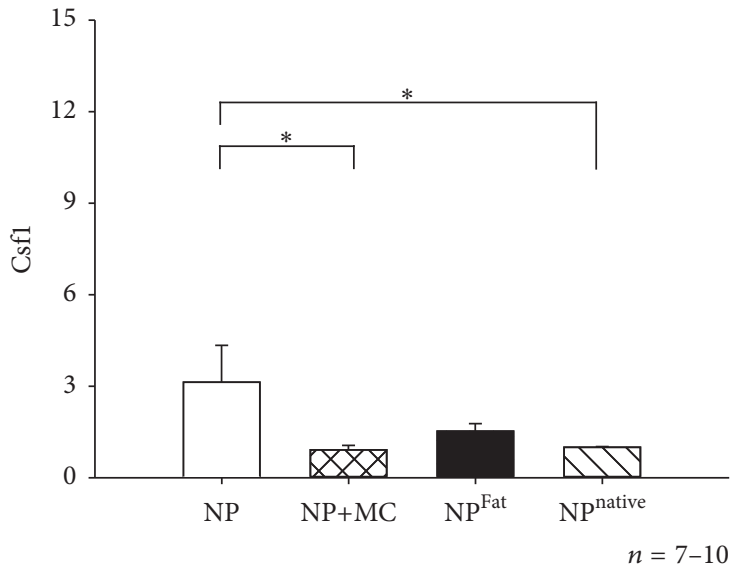

(c)

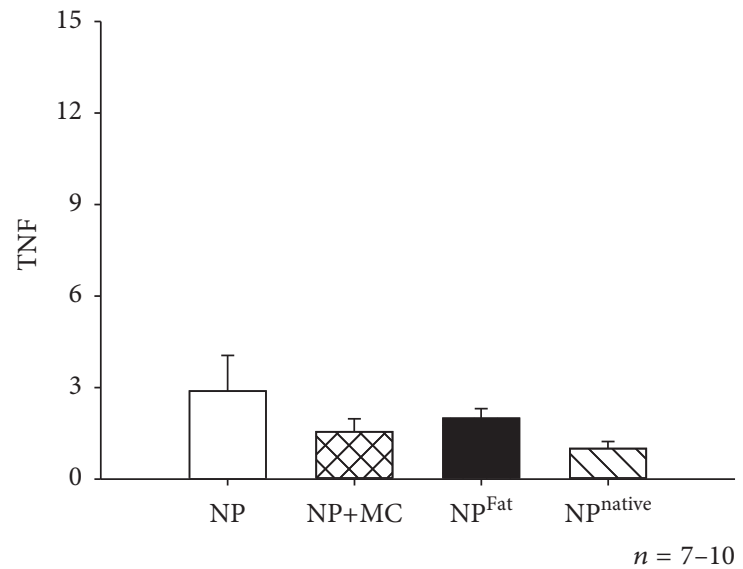

(b)

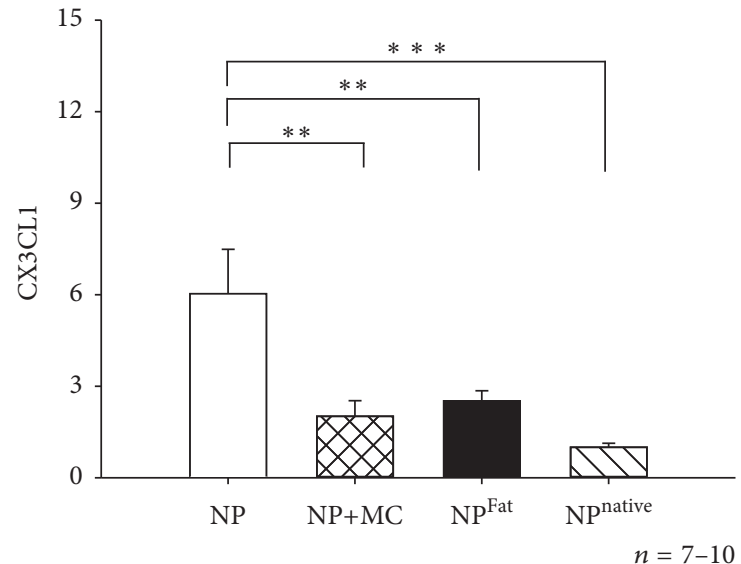

(d)

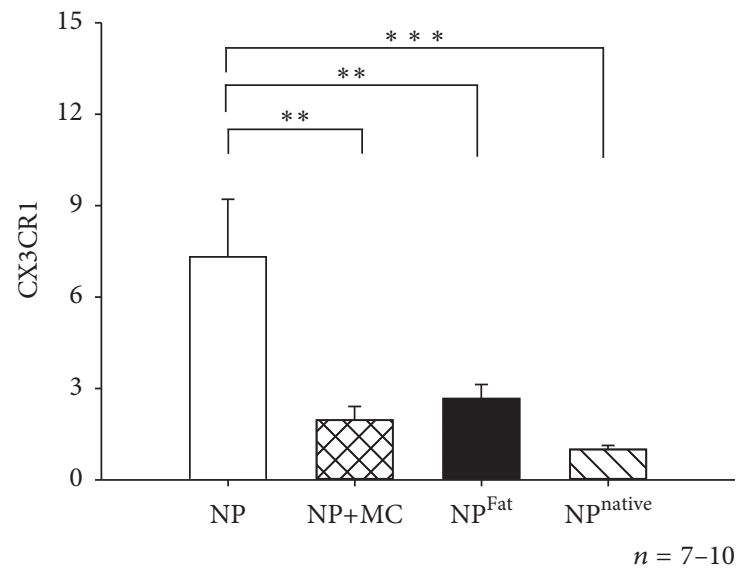

(e)

FIGURE 3: Fold expression of (a) IL-1 $\beta$, (b) TNF, (c) Csf1, (d) CX3CL1, and (e) CX3CR1 in NP in the three groups: NP in contact with dorsal nerve roots for 180 minutes (NP), NP in contact with dorsal nerve roots for 180 minutes together with minocycline (NP+MC), NP in contact with neck fat tissue for 180 minutes $\left(\mathrm{NP}^{\mathrm{Fat}}\right)$, and native nucleus pulposus $\left(\mathrm{NP}^{\text {native }}\right) .{ }^{*} P<0.05,{ }^{* *} P<0.01$, ${ }^{* * *} P<0.001$, one-way ANOVA, Tukey's post hoc test. 


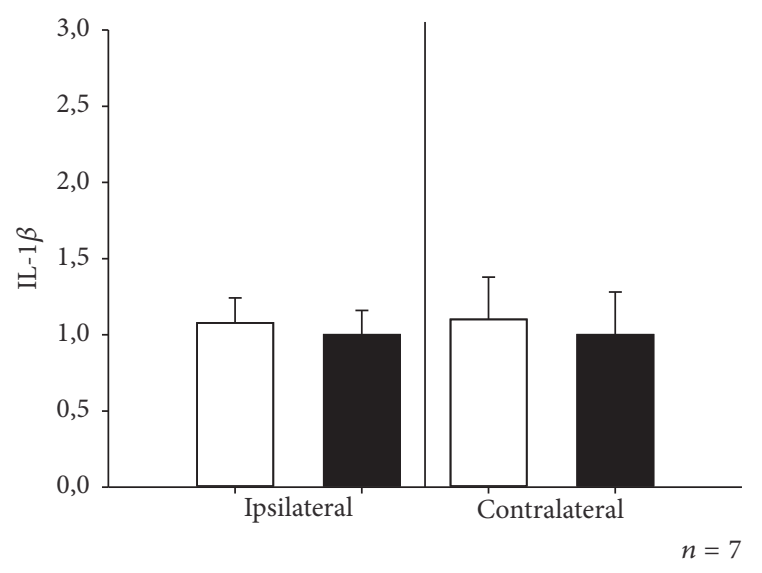

$\square \mathrm{NP}$

- Control

(a)

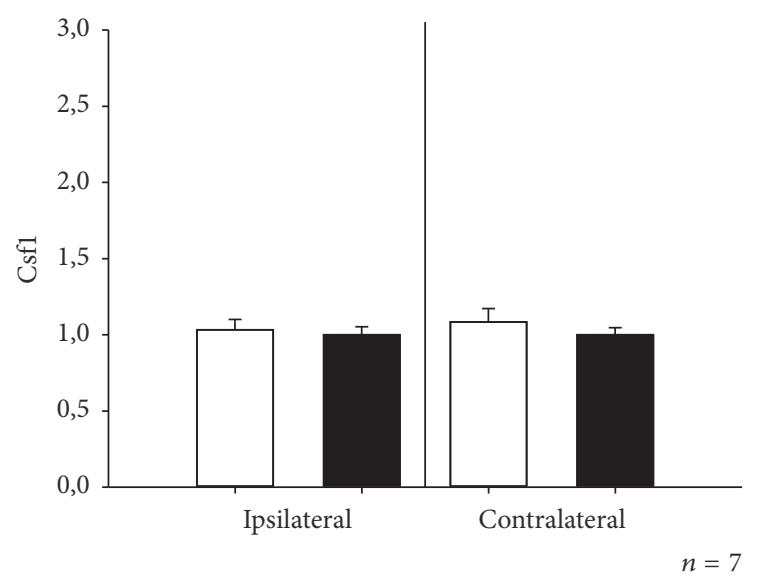

$\square \mathrm{NP}$

Control

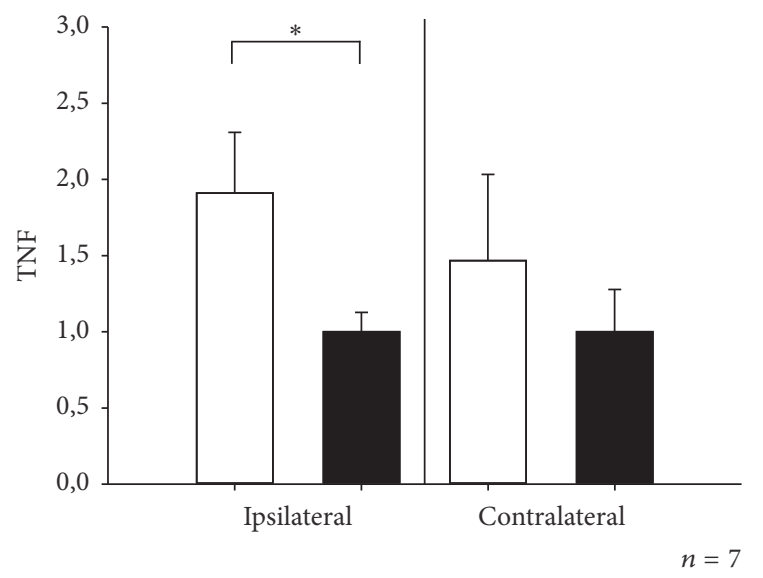

$\square \mathrm{NP}$

- Control

(b)

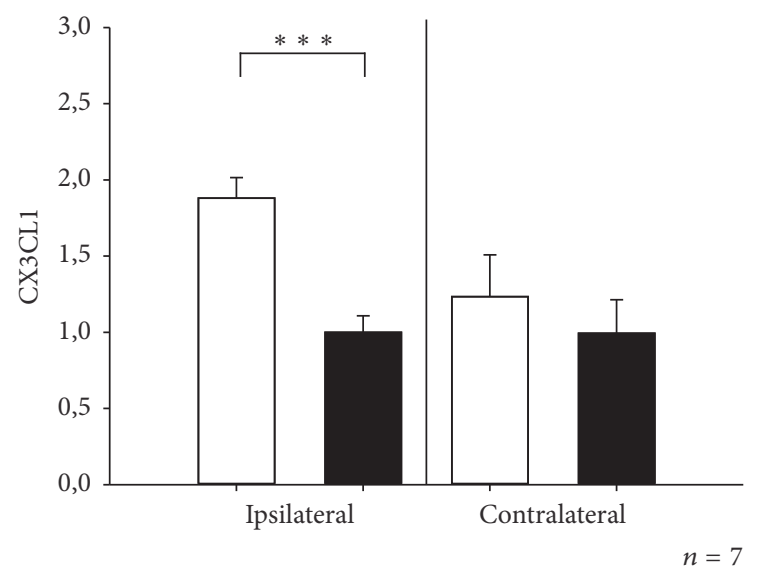

$\square$ NP

- Control

(c)

(d)

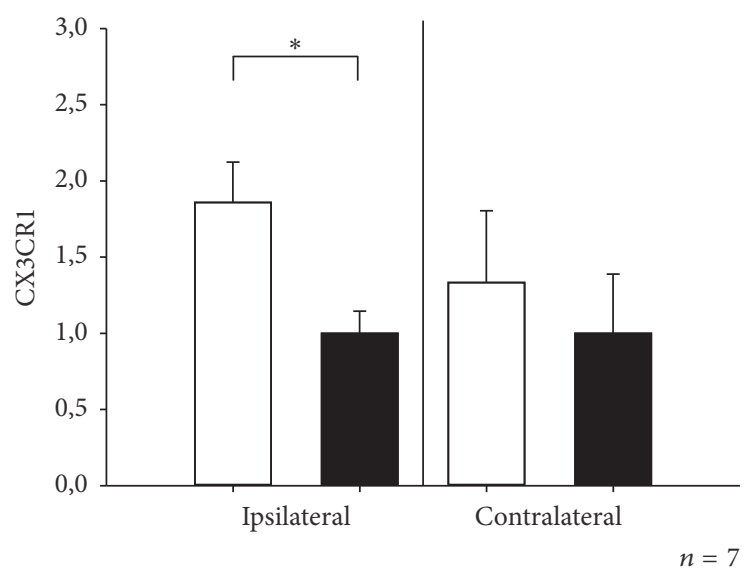

$\square$ NP

- Control

(e)

FIgURE 4: Fold expression of (a) IL-1 $\beta$, (b) TNF, (c) Csf1, (d) CX3CL1, and (e) CX3CR1 in the ipsi- and contralateral DRG after NP application onto the dorsal nerve roots. ${ }^{*} P<0.05,{ }^{* * *} P<0.001$, Student's $t$-test. 
observed in NP tissue following contact with intrascapular adipose tissue, suggesting a specific interaction between NP cells and neuronal tissue.

In accordance with the earlier observation that microglial inhibition may prevent hyperalgesia $[16,17,31]$, minocycline attenuated the increase in nociceptive signaling induced by NP in the present study. Data from earlier in vitro studies also suggest that inhibition of MAPK p38 in human NP cells inhibits inflammation [14]. Hence, it is tempting to speculate that the antinociceptive effects of minocycline may be a result of reduced MAPK p38 phosphorylation in microglia.

However, the anti-inflammatory effect of minocycline may be due to other mechanisms as well, not only by inhibition of $\mathrm{p} 38$ phosphorylation. For instance, minocycline is reportedly an inhibitor of nitric oxide synthase [32] and 5-lipoxygenase [33] attenuates TNF expression [34] and suppresses NF-kappa B activity [35]. In addition, minocycline may directly affect $\mathrm{Na}^{+}$-channels in primary afferent neurons [36], which could explain the short lasting drop in C-fiber response following minocycline administration. Minocycline also attenuated the increase in the gene expression of IL$1 \beta$, Csf1, CX3CL1, and CX3CR1 in NP tissue. The alleviating effect of minocycline on nociceptive signaling following application of NP onto the dorsal nerve roots may therefore be related to this anti-inflammatory processes in NP.

The changes in gene expression were less pronounced in the DRG than in the NP tissue itself. Still, a significant increase in the expression of TNF, CX3CL1, and CX3CR1 was demonstrated, supporting the previous observation that local application of NP onto the primary afferents may change the gene expression in the DRGs [37]. The changes in gene expression were only observed on the ipsilateral side. This supports the hypothesis that the observed nociceptive effect is dependent on contact between NP tissue and the dorsal nerve roots.

Increased expression of CX3CL1 and its receptor CX3CR1 has previously been shown in the DRG after nerve injury [38]. Our observation that hyperexcitability in the pain pathways after application of NP may involve an upregulation of CX3CL1 signaling in the DRG supports these previous findings. Moreover, evidence exists that CX3CL1 mediates inflammatory pain through activation of DRG satellite glial cells [7]. However, recent discoveries challenge the previous notion that CX3CL1 is expressed in DRG neurons [39]. Thus, it seems likely that the observed CX3CL1 upregulation takes place in endothelial cells, possibly facilitating infiltration of monocytes or other leukocytes into the DRG [40].

The distance from the NP graft to the DRG in our animal model may be larger than the distance from the NP to the DRG in lumbar disc herniation in patients. Still, our data show that application of NP onto the dorsal nerve roots induces a significant upregulation of several genes in the ipsilateral DRG tissue, emphasizing the clinical relevance of our animal model. Moreover, previous data show that peripheral inflammation through circulating IL- $1 \beta$ may increase the expression, of, for example, Cox 2, in the spinal cord [41]. Notably, circulating mediators released in the peripheral tissue may therefore also influence central secondary WDR neurons.

\section{Conclusion}

In summary, the present data suggest that hyperexcitability in the pain pathways and the local inflammation after disc herniation may involve upregulation of CX3CL1 signaling in both the NP and the DRG. The molecular consequence of the upregulation of CX3CL1 remains to be investigated.

\section{Ethical Approval}

All animal experiments were approved by the Norwegian Animal Research Authority and were performed in conformity with the laws and regulations controlling experiments and procedures on live animals in Norway.

\section{Competing Interests}

The authors declare that there are no competing interests regarding the publication of this paper.

\section{Authors' Contributions}

Daniel Pitz Jacobsen, Aurora Moen, Fred Haugen, and Johannes Gjerstad designed research; Daniel Pitz Jacobsen, Aurora Moen, Fred Haugen, and Johannes Gjerstad performed research; Daniel Pitz Jacobsen, Aurora Moen, and Johannes Gjerstad analyzed data; Aurora Moen and Fred Haugen contributed to the drafting of the manuscript; Daniel Pitz Jacobsen and Johannes Gjerstad wrote the paper.

\section{Acknowledgments}

The work was funded by the Norwegian Research Council (NFR).

\section{References}

[1] N. G. Egeland, A. Moen, L. M. Pedersen, H. Brisby, and J. Gjerstad, "Spinal nociceptive hyperexcitability induced by experimental disc herniation is associated with enhanced local expression of Csf1 and FasL," Pain, vol. 154, no. 9, pp. 1743-1748, 2013.

[2] J. M. Cuéllar, P. M. Borges, V. G. Cuéllar, A. Yoo, G. J. Scuderi, and D. C. Yeomans, "Cytokine expression in the epidural space: a model of noncompressive disc herniation-induced inflammation," Spine, vol. 38, no. 1, pp. 17-23, 2013.

[3] J. M. Cuellar, P. X. Montesano, and E. Carstens, "Role of TNF-alpha in sensitization of nociceptive dorsal horn neurons induced by application of nucleus pulposus to L5 dorsal root ganglion in rats," Pain, vol. 110, no. 3, pp. 578-587, 2004.

[4] N. J. Rothwell and P. J. L. M. Strijbos, "Cytokines in neurodegeneration and repair," International Journal of Developmental Neuroscience, vol. 13, no. 3-4, pp. 179-185, 1995.

[5] K. Olmarker, C. Nordborg, K. Larsson, and B. Rydevik, "Ultrastructural changes in spinal nerve roots induced by autologous nucleus pulposus," Spine, vol. 21, no. 4, pp. 411-414, 1996.

[6] K. Olmarker, B. Rydevik, and C. Nordborg, "Autologous nucleus pulposus induces neurophysiologic and histologic changes in porcine cauda equina nerve roots," Spine, vol. 18, no. 11, pp. 1425-1432, 1993. 
[7] G. R. Souza, J. Talbot, C. M. Lotufo, F. Q. Cunha, T. M. Cunha, and S. H. Ferreira, "Fractalkine mediates inflammatory pain through activation of satellite glial cells," Proceedings of the National Academy of Sciences of the United States of America, vol. 110, no. 27, pp. 11193-11198, 2013.

[8] D. E. Coyle, "Partial peripheral nerve injury leads to activation of astroglia and microglia which parallels the development of allodynic behavior," GLIA, vol. 23, no. 1, pp. 75-83, 1998.

[9] Z.-Y. Zhuang, P. Gerner, C. J. Woolf, and R.-R. Ji, "ERK is sequentially activated in neurons, microglia, and astrocytes by spinal nerve ligation and contributes to mechanical allodynia in this neuropathic pain model," Pain, vol. 114, no. 1-2, pp. 149-159, 2005.

[10] H. Ikeda, T. Kiritoshi, and K. Murase, "Contribution of microglia and astrocytes to the central sensitization, inflammatory and neuropathic pain in the juvenile rat," Molecular Pain, vol. 8, article 43, 2012.

[11] T. Imai, K. Hieshima, C. Haskell et al., "Identification and molecular characterization of fractalkine receptor CX3CR1, which mediates both leukocyte migration and adhesion," Cell, vol. 91, no. 4, pp. 521-530, 1997.

[12] A. Nishiyori, M. Minami, Y. Ohtani et al., "Localization of fractalkine and $\mathrm{CX}_{3} \mathrm{CR} 1 \mathrm{mRNAs}$ in rat brain: does fractalkine play a role in signaling from neuron to microglia?" FEBS Letters, vol. 429, no. 2, pp. 167-172, 1998.

[13] H. W. Park, S. H. Ahn, S. J. Kim et al., "Changes in spinal cord expression of fractalkine and its receptor in a rat model of disc herniation by autologous nucleus pulposus," Spine, vol. 36, no. 12, pp. E753-E760, 2011.

[14] R. K. Studer, A. M. Aboka, L. G. Gilbertson et al., "p38 MAPK inhibition in nucleus pulposus cells: a potential target for treating intervertebral disc degeneration," Spine, vol. 32, no. 25 , pp. 2827-2833, 2007.

[15] J. Huoyan, W. Huimin, Z. Fupeng, L. Xiaohong, X. Lu, and A. Shen, "PPAR $\gamma$ agonist pioglitazone inhibits microglia inflammation by blocking p38 mitogen-activated protein kinase signaling pathways," Inflammation Research, vol. 59, no. 11, pp. 921-929, 2010.

[16] A. Ledeboer, E. M. Sloane, E. D. Milligan et al., "Minocycline attenuates mechanical allodynia and proinflammatory cytokine expression in rat models of pain facilitation," Pain, vol. 115, no. 1-2, pp. 71-83, 2005.

[17] X. Zhang, Y. Xu, J. Wang et al., "The effect of intrathecal administration of glial activation inhibitors on dorsal horn BDNF overexpression and hind paw mechanical allodynia in spinal nerve ligated rats," Journal of Neural Transmission, vol. 119, no. 3, pp. 329-336, 2012.

[18] S. A. Malin, B. M. Davis, and D. C. Molliver, "Production of dissociated sensory neuron cultures and considerations for their use in studying neuronal function and plasticity," Nature Protocols, vol. 2, no. 1, pp. 152-160, 2007.

[19] L. M. Pedersen, L. M. Jacobsen, S. Mollerup, and J. Gjerstad, "Spinal cord long-term potentiation (LTP) is associated with increased dorsal horn gene expression of IL-1 $\beta$, GDNF and iNOS," European Journal of Pain, vol. 14, no. 3, pp. 255-260, 2010.

[20] H. Brisby and I. Hammar, "Thalamic activation in a disc herniation model," Spine, vol. 32, no. 25, pp. 2846-2852, 2007.

[21] Y. Kawasaki, L. Zhang, J.-K. Cheng, and R.-R. Ji, "Cytokine mechanisms of central sensitization: distinct and overlapping role of interleukin- $\beta$, interleukin- 6 , and tumor necrosis factor$\alpha$ in regulating synaptic and neuronal activity in the superficial spinal cord," Journal of Neuroscience, vol. 28, no. 20, pp. 51895194,2008

[22] Z.-Y. Zhuang, Y. Kawasaki, P.-H. Tan, Y.-R. Wen, J. Huang, and R.-R. Ji, "Role of the CX3CR1/p38 MAPK pathway in spinal microglia for the development of neuropathic pain following nerve injury-induced cleavage of fractalkine," Brain, Behavior, and Immunity, vol. 21, no. 5, pp. 642-651, 2007.

[23] K. Olmarker and K. Larsson, "Tumor necrosis factor $\alpha$ and nucleus-pulposus-induced nerve root injury," Spine, vol. 23, no. 23, pp. 2538-2544, 1998.

[24] K. Y. Yang, W. S. Bae, M. J. Kim et al., "Participation of the central p38 and ERK1/2 pathways in IL-1beta-induced sensitization of nociception in rats," Progress in Neuro-Psychopharmacology \& Biological Psychiatry, vol. 46, pp. 98-104, 2013.

[25] X.-H. Wei, T. Yang, Q. Wu et al., "Peri-sciatic administration of recombinant rat IL- $1 \beta$ induces mechanical allodynia by activation of src-family kinases in spinal microglia in rats," Experimental Neurology, vol. 234, no. 2, pp. 389-397, 2012.

[26] B. Shafit-Zagardo, N. Sharma, J. W. Herman, M. B. Bornstein, and C. F. Brosnan, "CSF-1 expression is upregulated in astrocyte cultures by IL- 1 and TNF and affects microglial proliferation and morphology in organotypic cultures," International Journal of Developmental Neuroscience, vol. 11, no. 2, pp. 189-198, 1993.

[27] G. Gowing, M. Lalancette-Hébert, J.-N. Audet, F. Dequen, and J.-P. Julien, "Macrophage colony stimulating factor (MCSF) exacerbates ALS disease in a mouse model through altered responses of microglia expressing mutant superoxide dismutase," Experimental Neurology, vol. 220, no. 2, pp. 267-275, 2009.

[28] M. Sawada, A. Suzumura, H. Yamamoto, and T. Marunouchi, "Activation and proliferation of the isolated microglia by colony stimulating factor-1 and possible involvement of protein kinase C," Brain Research, vol. 509, no. 1, pp. 119-124, 1990.

[29] M. Kiyomoto, M. Shinoda, A. Okada-Ogawa et al., "Fractalkine signaling in microglia contributes to ectopic orofacial pain following trapezius muscle inflammation," The Journal of Neuroscience, vol. 33, no. 18, pp. 7667-7680, 2013.

[30] A. K. Clark, A. A. Staniland, and M. Malcangio, "Fractalkine/ CX3CR1 signalling in chronic pain and inflammation," Current Pharmaceutical Biotechnology, vol. 12, no. 10, pp. 1707-1714, 2011.

[31] C.-S. Sung, C.-H. Cherng, Z.-H. Wen et al., "Minocycline and fluorocitrate suppress spinal nociceptive signaling in intrathecal IL- $1 \beta$-induced thermal hyperalgesic rats," Glia, vol. 60 , no. 12 , pp. 2004-2017, 2012.

[32] A. R. Amin, M. G. Attur, G. D. Thakker et al., "A novel mechanism of action of tetracyclines: effects on nitric oxide synthases," Proceedings of the National Academy of Sciences of the United States of America, vol. 93, no. 24, pp. 14014-14019, 1996.

[33] Y. Song, E.-Q. Wei, W.-P. Zhang, L. Zhang, J.-R. Liu, and Z. Chen, "Minocycline protects PC12 cells from ischemic-like injury and inhibits 5-lipoxygenase activation," NeuroReport, vol. 15, no. 14, pp. 2181-2184, 2004.

[34] S. M. Lee, T. Y. Yune, S. J. Kim et al., "Minocycline inhibits apoptotic cell death via attenuation of TNF- $\alpha$ expression following iNOS/NO induction by lipopolysaccharide in neuron/glia cocultures," Journal of Neurochemistry, vol. 91, no. 3, pp. 568-578, 2004.

[35] P. Ataie-Kachoie, S. Badar, D. L. Morris, and M. H. Pourgholami, "Minocycline targets the NF- $\kappa \mathrm{B}$ nexus through suppression of TGF- $\beta 1$-TAK1-I $\kappa \mathrm{B}$ signaling in ovarian cancer," Molecular Cancer Research, vol. 11, no. 10, pp. 1279-1291, 2013. 
[36] T. H. Kim, H. I. Kim, J. Kim, M. Park, and J.-H. Song, "Effects of minocycline on $\mathrm{Na}^{+}$currents in rat dorsal root ganglion neurons," Brain Research, vol. 1370, pp. 34-42, 2011.

[37] K. Takahashi Sato, K. Satoh, M. Sekiguchi et al., "Local application of nucleus pulposus induces expression OF P2X3 in rat dorsal root ganglion cells," Fukushima Journal of Medical Science, vol. 58, no. 1, pp. 17-21, 2012.

[38] W. Zhu, C. Acosta, B. Macneil et al., "Elevated expression of fractalkine (CX3CL1) and fractalkine receptor (CX3CR1) in the dorsal root ganglia and spinal cord in experimental autoimmune encephalomyelitis: implications in multiple sclerosisinduced neuropathic pain," BioMed Research International, vol. 2013, Article ID 480702, 14 pages, 2013.

[39] K.-W. Kim, A. Vallon-Eberhard, E. Zigmond et al., "In vivo structure/function and expression analysis of the CX3C chemokine fractalkine," Blood, vol. 118, no. 22, pp. e156-e167, 2011.

[40] E. A. Old, S. Nadkarni, J. Grist et al., "Monocytes expressing CX3CR1 orchestrate the development of vincristine-induced pain," The Journal of Clinical Investigation, vol. 124, no. 5, pp. 2023-2036, 2014.

[41] T. A. Samad, K. A. Moore, A. Sapirstein et al., "Interleukin$1 \beta$-mediated induction of Cox- 2 in the CNS contributes to inflammatory pain hypersensitivity," Nature, vol. 410, no. 6827, pp. 471-475, 2001. 


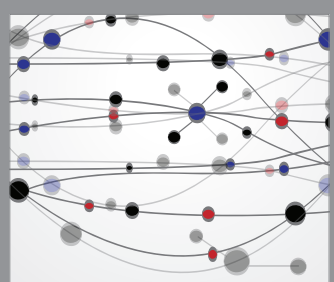

The Scientific World Journal
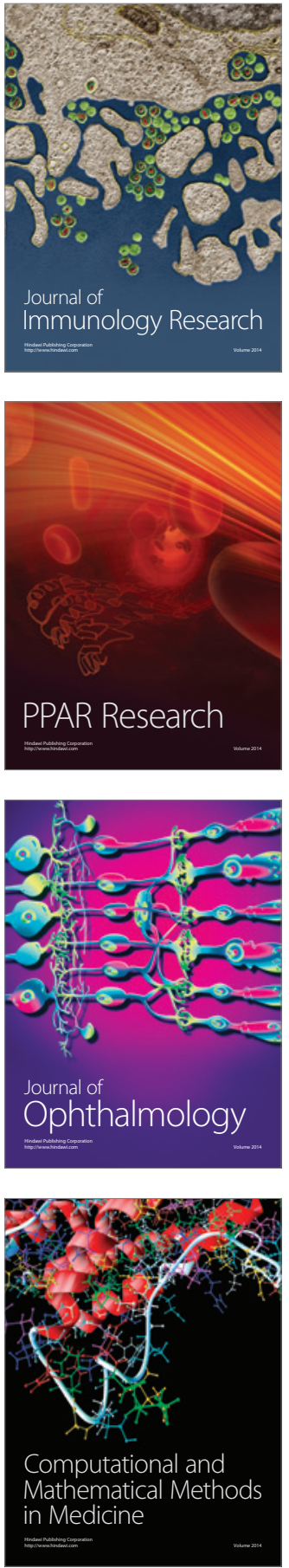

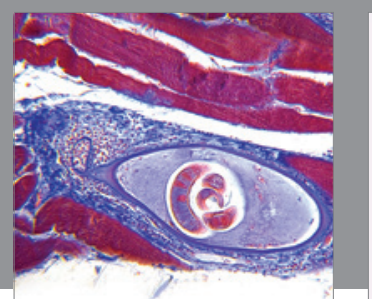

Gastroenterology Research and Practice

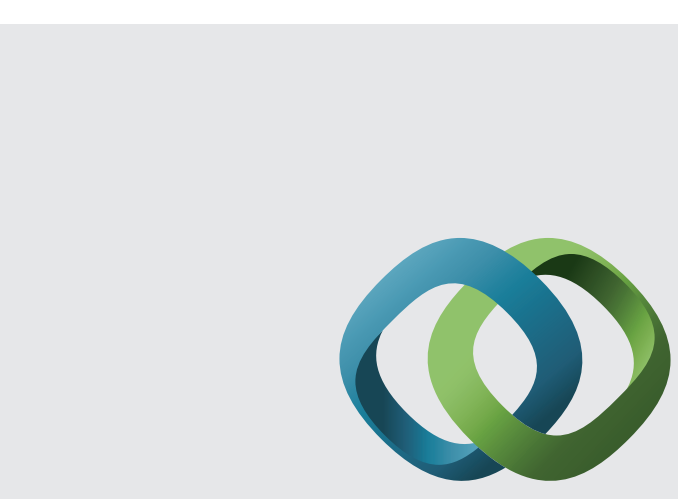

\section{Hindawi}

Submit your manuscripts at

http://www.hindawi.com
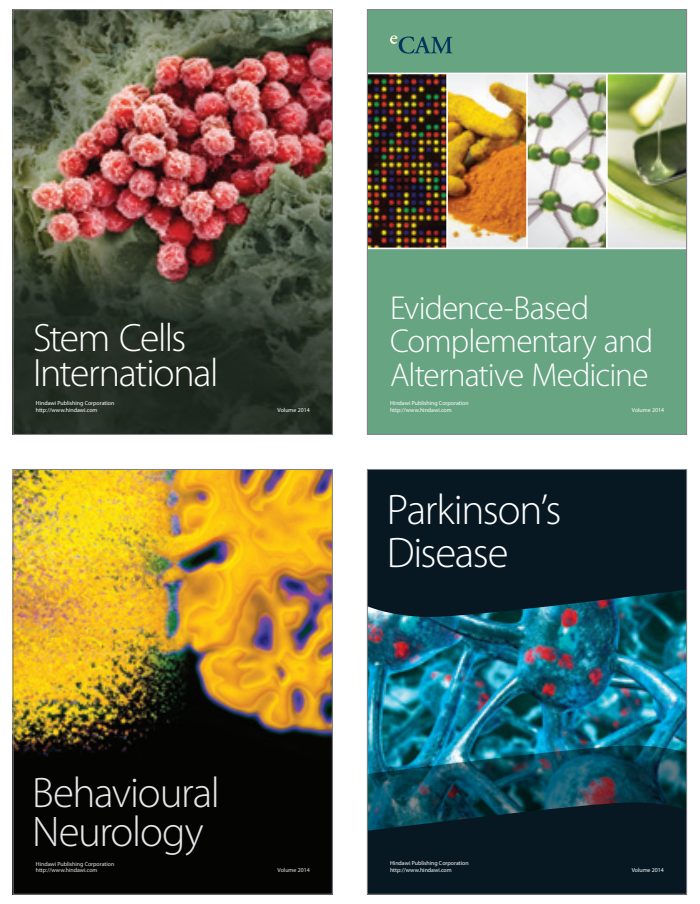
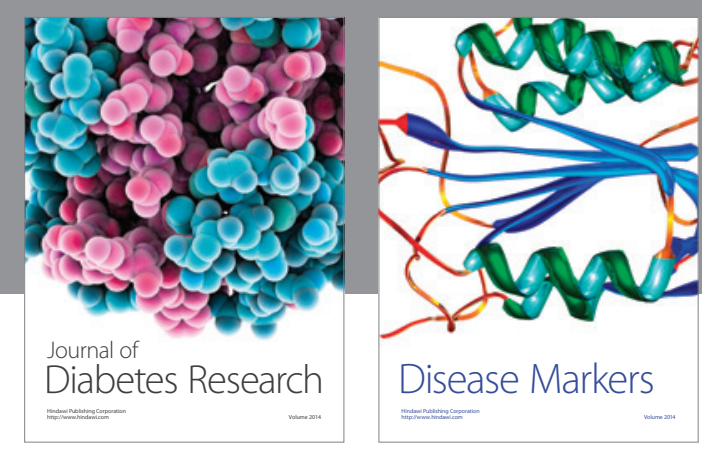

Disease Markers
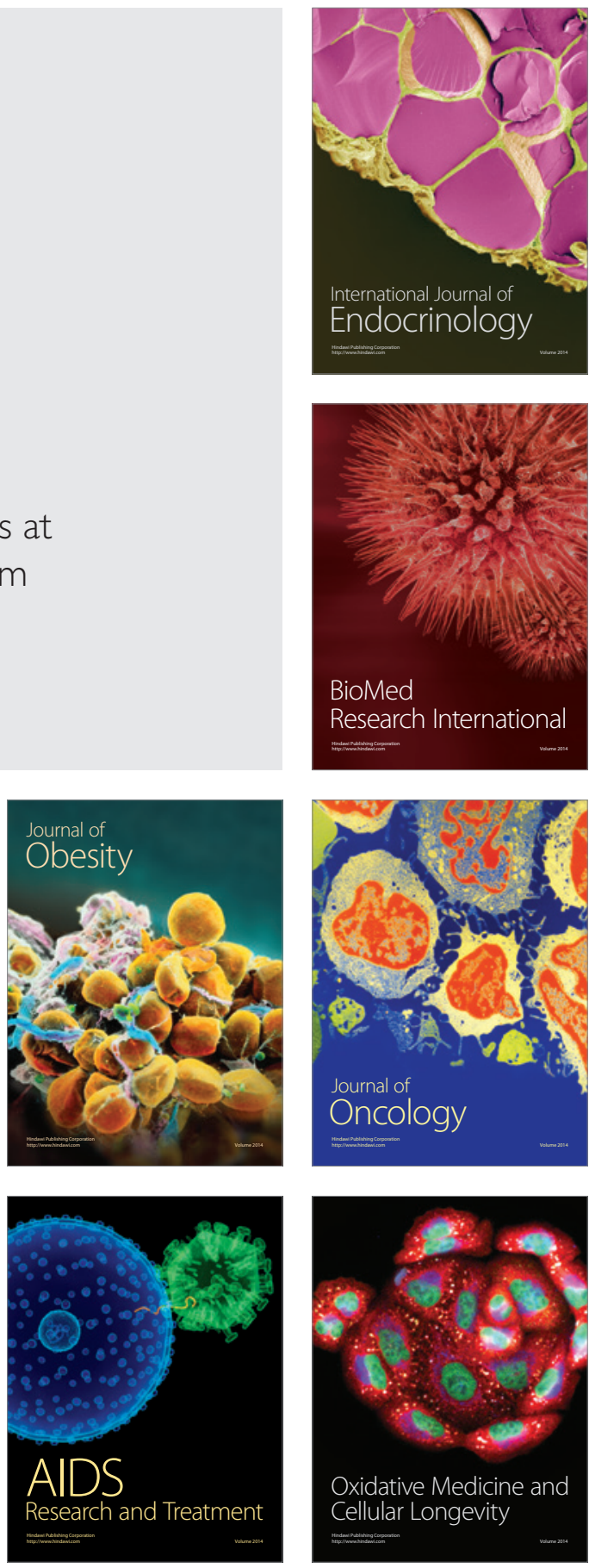